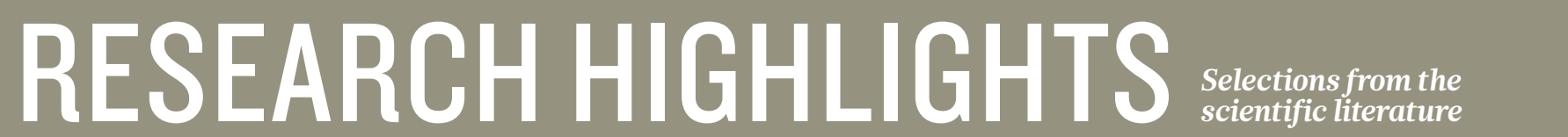

CHEMISTRY

\section{Zinc can swim in an electric field}

A tiny zinc object that can propel itself from one end of a capillary tube to the other holds promise for those striving to make minuscule motors.

Gabriel Loget and Alexander Kuhn at the University of Bordeaux in France immersed their micrometre-sized zinc 'swimmer' in a solution of zinc sulphate and applied a relatively weak external electric field. The field promotes oxidation on one side of the swimmer, dissolving the zinc, and reduction on the other, which deposits zinc, thus moving the object along. The swimmer reached speeds of 80 micrometres per second.

The authors suggest that their method could be an efficient way to control the motion of metallic microobjects in engineered micromotors - or perhaps even nanomotors.

J. Am. Chem. Soc. doi:10.1021/ ja107644x (2010)

\section{GLACIOLOGY}

\section{Heat trickles} down in ice sheets

Meltwater is thought to be contributing to accelerated glacier flow. Thomas Phillips and his colleagues at the University of Colorado at

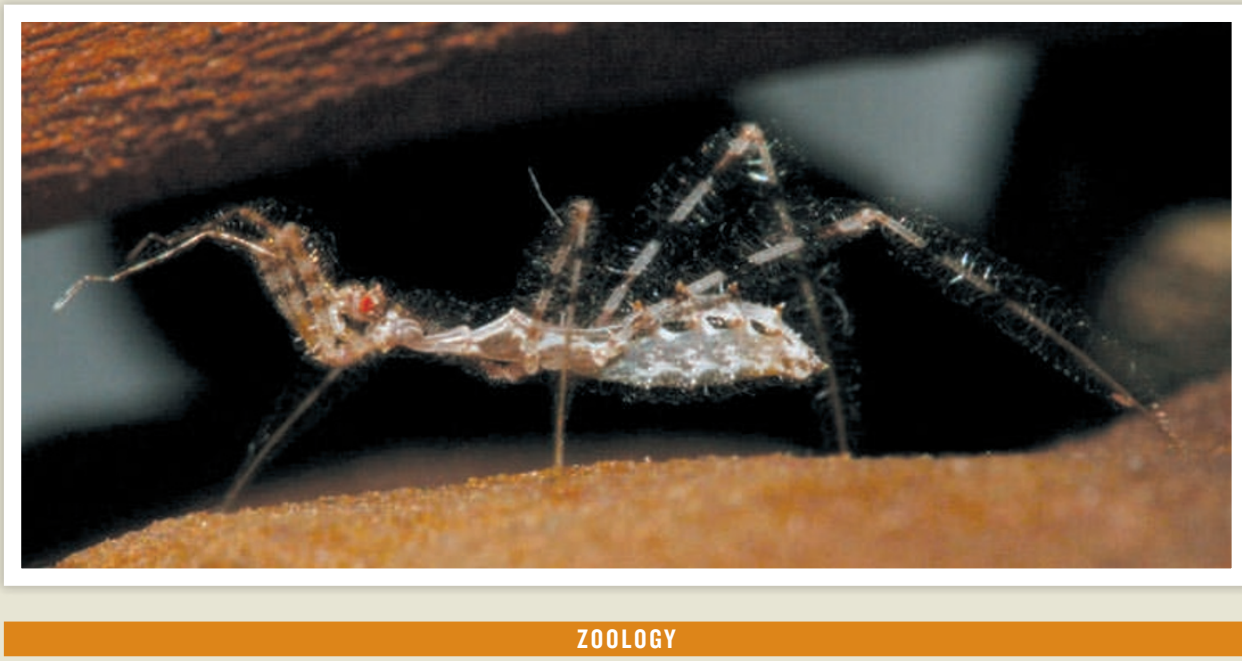

\section{Spiders lured by subterfuge}

Assassin bugs hunt spiders by plucking the threads of spiderwebs to draw the arachnids closer. Researchers in Australia report that the vibrations mimic those generated by insects trapped in the webs - and are even at specific frequencies to avoid eliciting an aggressive response.

In a series of laboratory tests, Anne Wignall and Phillip Taylor of Macquarie University in Sydney show that the vibrations induced by
Stenolemus bituberus (pictured) trigger the same behaviour from spiders as do those of some prey species. Vibrations from falling leaves elicited no response, whereas those from courting male spiders led females to adopt copulatory positions.

The assassin bugs seem to avoid producing the higher frequency vibrations generated by some prey that can trigger a dangerous highspeed approach from the spider.

Proc. R. Soc. B doi:10.1098/rspb.2010.2060 (2010)

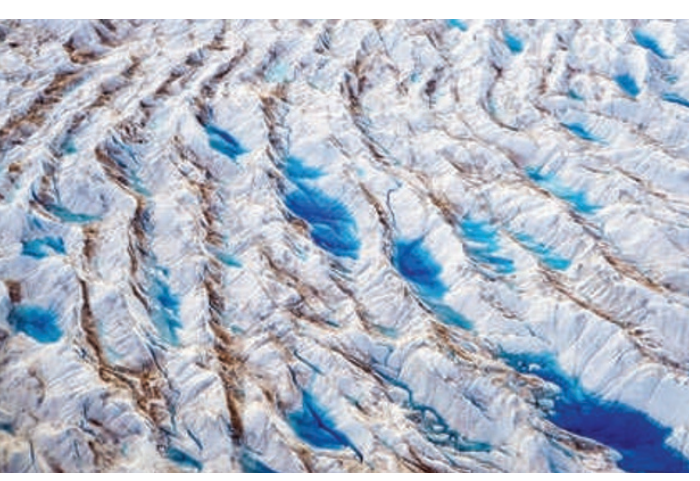

Boulder show that meltwater seeping through fractures and crevasses in the ice (pictured) can have a sustained warming effect, speeding up ice flow.

By incorporating the effect of this seeping water into thermal models of ice sheets, the authors found that the relatively temperate meltwater warms the surrounding ice, thereby reducing its viscosity and helping to increase its flow. Most of the water refreezes during the winter, but small quantities can remain liquid, causing the sustained warming.

This effect could be leading to much faster glacial responses to warming than current models predict. It is probably contributing to observed changes in some regions of the Greenland ice sheet, the authors suggest.

Geophys. Res. Lett. doi:10.1029/ 2010GL044397 (2010)

\section{CANCER BIOLOGY}

\section{Cell neighbours aid cancer relapse}

Cancer patients often relapse after seemingly successful chemotherapy - perhaps because cellular stress responses to the drugs shield some cancer cells, creating a protected reservoir that can seed fresh tumour growth.

Luke Gilbert and Michael
Hemann at the Massachusetts Institute of Technology in Cambridge studied the environment surrounding tumour cells in a mouse model of Burkitt's lymphoma, a cancer of the lymphatic system. They found that normal cells in the thymus, an organ that consists mainly of lymphatic tissue, release two proteins in response to the DNA damage caused by chemotherapy. Those proteins, IL-6 and Timp1 , then protect nearby lymphoma cells from the lethal effects of the drugs.

The same thing happened in human liver-cancer cells grown in culture and treated with doxorubicin, a common treatment for liver cancer. Cell 143, 355-366 (2010) 